\title{
Erratum: Canonical Hamiltonian for an extended test body in curved spacetime: To quadratic order in spin [Phys. Rev. D 93, 103008 (2016)]
}

\author{
Justin Vines@, Daniela Kunst, Jan Steinhoff, and Tanja Hinderer
}

(Q) (Received 28 June 2021; published 28 July 2021)

DOI: $10.1103 /$ PhysRevD.104.029902

We correct errors in the published versions of Eqs. (D7) and (D8), giving the test-spin-squared contributions to the Hamiltonian to all PN orders (for zero Kerr spin). These were copy errors only in Eqs. (D7) and (D8) which did not affect the comparisons to PN results in Sec. VII C. We have checked that the new expressions below, replacing Eqs. (D7) and (D8), along with (D1)-(D6), expanded to the appropriate orders, are in complete agreement (modulo a canonical transformation) with the extended test-body limits of both the second post-Minkowskian spin-sqaured results of Kosmopoulos and Luna [1] and the next-to-next-to-leading-order post-Newtonian (4PN) spin-squared results of Levi and Steinhoff [2].

The corrected replacements for Eqs. (D7) and (D8) read

$$
H_{S^{2}}=\vec{S}^{2} k_{1}(\vec{z}, \vec{P})+(\vec{n} \cdot \vec{S})^{2} k_{2}(\vec{z}, \vec{P})+(\vec{P} \cdot \vec{S})^{2} k_{3}(\vec{z}, \vec{P})+\vec{n} \cdot \vec{P} \vec{n} \cdot \vec{S} \vec{P} \cdot \vec{S} k_{4}(\vec{z}, \vec{P})
$$

with

$$
\begin{aligned}
k_{1}= & \frac{M}{(\hat{Q}+m) r^{3}}+\frac{(1-\sqrt{w})^{2} r-2 M}{2 \hat{Q} r^{3}} \sqrt{w}+\left(\frac{M(2+\sqrt{w})}{2 \hat{Q}(\hat{Q}+m)^{2} r^{3}}+\frac{(1-\sqrt{w})^{2}}{2 \hat{Q}^{3} r^{2}} \sqrt{w}\right)\left((\vec{n} \cdot \vec{P})^{2}-\vec{P}^{2}\right) \\
& -(C-1) \frac{M \sqrt{w}}{2 m^{2} \hat{Q} r^{3}}\left(m^{2}-3(\vec{n} \cdot \vec{P})^{2}+3 \vec{P}^{2}\right), \\
k_{2}= & M \frac{(\hat{Q}+3 m) \sqrt{w}-\hat{Q}}{\hat{Q}(\hat{Q}+m) r^{3}}-\frac{(1-\sqrt{w})^{2}}{2 \hat{Q} r^{2}} \sqrt{w}+\frac{M \sqrt{w}(w-2+\sqrt{w})}{\hat{Q}(\hat{Q}+m)^{2} r^{3}}(\vec{n})^{2}+\left(\frac{M(1+2 \sqrt{w})}{\hat{Q}(\hat{Q}+m)^{2} r^{3}}+\frac{(1-\sqrt{w})^{2}}{2 \hat{Q}^{3} r^{2}} \sqrt{w}\right) \vec{P}^{2} \\
& +(C-1) \frac{3 M \sqrt{w}}{m^{2} r^{3}}\left(\frac{\hat{Q}^{2}+\vec{P}^{2}}{2 \hat{Q}}+\frac{1-\sqrt{w}}{\hat{Q}+m} \sqrt{w}(\vec{n} \cdot \vec{P})^{2}+\frac{(1-\sqrt{w})^{2}}{2 \hat{Q}(\hat{Q}+m)^{2}} w(\vec{n} \cdot \vec{P})^{4}\right), \\
k_{3}= & \frac{M}{\hat{Q}(\hat{Q}+m)^{2} r^{3}}+\frac{(1-\sqrt{w})^{2}}{2 \hat{Q}^{3} r^{2}} \sqrt{w}+(C-1) \frac{3 M \sqrt{w}}{2 m^{2} \hat{Q} r^{3}}\left(1+w \frac{(\vec{n} \cdot \vec{P})^{2}}{(\hat{Q}+m)^{2}}\right), \\
k_{4}= & -\frac{M(2+w)}{\hat{Q}(\hat{Q}+m)^{2} r^{3}}-\frac{(1-\sqrt{w})^{2}}{\hat{Q}^{3} r^{2}} \sqrt{w}-(C-1) \frac{3 M \sqrt{w}}{m^{2} r^{3}}\left(\frac{1}{\hat{Q}}+\frac{\sqrt{w}}{\hat{Q}+m}+w(1-\sqrt{w}) \frac{(\vec{n} \cdot \vec{P})^{2}}{\hat{Q}(\hat{Q}+m)^{2}}\right) .
\end{aligned}
$$

We are grateful to Dimitrios Kosmopoulos and Andrés Luna for pointing us toward these errors.

[1] D. Kosmopoulos and A. Luna, Quadratic-in-spin Hamiltonian at $\mathcal{O}\left(G^{2}\right)$ from scattering amplitudes, arXiv:2102.10137.

[2] M. Levi and J. Steinhoff, Complete conservative dynamics for inspiralling compact binaries with spins at fourth post-Newtonian order, arXiv:1607.04252. 